CrossMark \&click for updates

Cite this: Phys. Chem. Chem. Phys., 2016, 18, 5299

Received 7th October 2015, Accepted 20th January 2016

DOI: $10.1039 / c 5 c p 06011 \mathrm{~g}$

www.rsc.org/pccp

\section{Optical and morphological properties of thin films of bis-pyrenyl $\pi$-conjugated molecules $\dagger$}

\author{
Tony Lelaidier, ${ }^{a b}$ Tobias Lünskens, ${ }^{a}$ Alexander von Weber, ${ }^{a}$ Thomas Leoni, ${ }^{b}$ \\ Alain Ranguis, ${ }^{\mathrm{b}}$ Anthony D'Aléo, ${ }^{\mathrm{b}}$ Frédéric Fages, ${ }^{\mathrm{b}}$ Aras Kartouzian, ${ }^{\mathrm{a}}$ Conrad Becker ${ }^{\mathrm{b}}$ \\ and Ulrich Heiz ${ }^{a}$
}

1,4-Di-n-octyloxy-2,5-bis(pyren-1-ylethenyl)benzene (bis-pyrene) has been studied by the means of surface cavity ring-down (s-CRD) spectroscopy on an amorphous BK7 glass substrate and scanning tunnelling microscopy (STM) on Au(111). Absorption spectra show a modification of the optical properties as a function of coverage, i.e. appearance of a shoulder around $505 \mathrm{~nm}$ followed by a saturation of the intensity of this signal observed at higher coverages. We attribute this shoulder to the change of the molecular orientation between the first and the second monolayer and thus to an interfacial effect. These results are confirmed by scanning tunnelling microscopy (STM) measurements where the bis-pyrene molecules have been deposited on $\mathrm{Au}(111)$ at room temperature (RT) and onto a cold substrate. Independently of the temperature in the range from $210 \mathrm{~K}$ to RT, the first monolayer is always highly organized. At low temperature bis-pyrene molecules constituting the second monolayer are randomly distributed, suggesting that self-organisation is kinetically hindered. Deposited at room temperature, the molecular diffusion is enhanced and the formation of an organized second layer takes place after storing the sample for 150 minutes at room temperature. A HOMO-LUMO gap of $2.85 \mathrm{eV}$ has been determined by scanning tunnelling spectroscopy, which is in very good agreement with the observed optical transition at $434 \mathrm{~nm}(2.86 \mathrm{eV})$ in s-CRD spectroscopy.

\section{Introduction}

These days $\pi$-conjugated materials continue to receive considerable attention because they combine interesting electrical and optical properties and easy processability, making them key materials for advanced applications in organic field effect transistors (OFETs), organic photovoltaic (OPV) cells, and organic light emitting diodes (OLEDs). The insertion of pyrene groups into conjugated oligomers results in highly conjugated structures with unique photo-physical properties. ${ }^{1-5}$ The comprehension and ultimately the control at the nanoscale of organic-organic and organic-inorganic interfaces pertaining to these devices are of paramount importance in order to ensure performant device functions. ${ }^{6}$ It is wellestablished that the molecular adsorption geometry not only influences the growth of the subsequent layers, but also has a strong impact on both the charge carrier transport in the organic thin films and charge carrier injection processes at

\footnotetext{
${ }^{a}$ Technische Universität München, Department of Physical Chemistry, Catalysis Research Center, Lichtenbergstr. 4, 85748 Garching, Germany

${ }^{b}$ Aix-Marseille Université, CNRS, CINAM UMR 7325, 13288 Marseille, France. E-mail: lelaidier@cinam.univ-mrs.fr; Fax: +33 (0)491418916; Tel: +33 (0) 491172800

$\dagger$ Electronic supplementary information (ESI) available. See DOI: 10.1039/c5cp06011g
}

metallic contacts. Therefore the understanding of organic thin film growth is mandatory to improve and control devices.

In this study, we describe a high-resolution study of thin films of a symmetrical bis-pyrenyl compound, bis-pyrene, (Fig. 1), in which a bis(vinylene)-phenylene unit bridges two pyrene rings. Pyrene-containing conjugated materials are known to possess versatile electronic and optical properties. ${ }^{7,8}$ Preliminary results showed that bis-pyrene behaves as p-type organic semiconductor. ${ }^{9}$ Owing to its extended $\pi$-conjugated structures, we show that bis-pyrene displays optical properties that allowed the use of s-cavity ring-down (CRD) spectroscopy. s-CRD is a direct

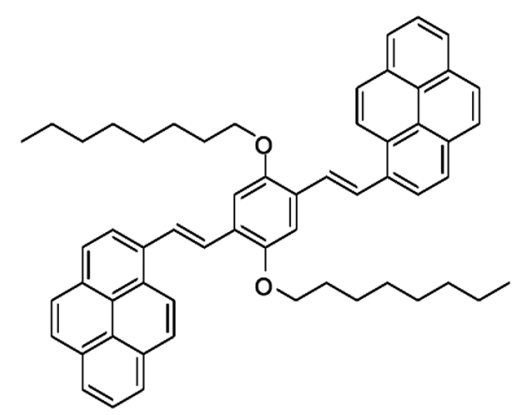

Fig. 1 Chemical structure of the bis-pyrene compound. 
absorption technique, which permits the investigation of the optical properties of surface adsorbates at extremely low coverages down to $1 \%$ of a monolayer. ${ }^{10,11}$

CRD spectroscopy owes its sensitivity mainly to the following two factors. Firstly, in contrast to other absorption methods, it measures the time constant of the optical cavity containing the sample instead of changes in the light intensity. Secondly, the effective path length through the sample is greatly enhanced (many thousands times) by the cavity. ${ }^{10-12}$ The main advantage of s-CRD spectroscopy compared to highly surface sensitive nonlinear technique lies in the unambiguousness of spectral assignments $^{13,14}$ and the ease of reference measurements. ${ }^{15}$ Here we combine s-CRD spectroscopy and scanning tunnelling microscopy (STM) to study thin bis-pyrene films in the coverage range from 0.2 to 4 monolayers (ML), in order to monitor their optical and morphological properties during the growth process.

\section{Synthesis}

The bis-pyrenyl compound bis-pyrene was synthesized in one step using a Wittig reaction. To a suspension of the bis(triphenylphosphonium) derivative of 1,4-bis(bromomethyl)2,5-bis(n-octyloxy)benzene16 (1.00 g, $1 \mathrm{mmol})$ in freshly distilled THF $(20 \mathrm{~m}), 95 \%$ $\mathrm{NaH}$ ( $0.10 \mathrm{~g}, 4 \mathrm{mmol})$ was added portion-wise at room temperature. A solution of the commercially available pyrene-1-carbaldehyde $(0.46 \mathrm{~g}, 2 \mathrm{mmol})$ in THF $(10 \mathrm{~mL})$ was added. The reaction mixture was heated overnight at $30{ }^{\circ} \mathrm{C}$, then cooled down to room temperature prior to solvent evaporation. The residue was dissolved in dichloromethane. The organic layer was washed with water and then dried over $\mathrm{Na}_{2} \mathrm{SO}_{4}$. The solvent was removed under vacuum and the crude product was subjected to column chromatography (silica gel) eluting with dichloromethane then crystallized from a mixture of dichloromethane-methanol. Compound bis-pyrene was obtained ( $0.47 \mathrm{~g}, 60 \%$ yield) as an orange solid. The all-trans configuration was confirmed by ${ }^{1}$ H NMR (see ESI, $\dagger$ Fig. S1).

\section{Experimental}

The s-CRD setup is constituted of a picosecond laser system, an ultrahigh vacuum (UHV) analysis chamber, a high vacuum (HV) preparation chamber, a photomultiplier tube and an oscilloscope, which have been described in detail elsewhere. ${ }^{10,15,16}$ For each experiment commercially available BK7 amorphous glass substrates were first cleaned carefully with spectroscopic grade acetone and then introduced into the preparation chamber via a fast entry lock. The sample was cooled down to $120 \mathrm{~K}$ (temperature measured at the glass substrate using a thermocouple) prior to argon ion sputtering. The bis-pyrene molecules were evaporated onto the cold substrate under HV conditions. A quartzmicro-balance was used for the determination of the molecular density from the frequency shift using Sauerbrey's equation. Inside the analysis chamber, four pairs of highly reflective mirrors mounted onto a pair of UHV compatible mirror exchangers ${ }^{10}$ were used to cover the spectral range from 420 to $680 \mathrm{~nm}$.
Overlapping wavelength regions of at least $20 \mathrm{~nm}$ were available allowing for the comparison of spectra recorded by cavities with adjacent wavelengths. All measurements were performed at Brewster's angle in order to reduce the loss induced by reflection. ${ }^{10-12}$ The light leaving the optical cavity was detected by the means of a photomultiplier tube and recorded by an oscilloscope. For a better signal/noise ratio, 100 ring-down signals were averaged for each wavelength. More information concerning the data acquisition and data treatment processes are available in the ESI. $\dagger$

All STM experiments and sample preparations were performed under UHV conditions, using a commercial low-temperature scanning tunnelling microscope (LT-STM). The LT-STM was used at liquid nitrogen temperature (both sample and tip) for all experiments presented here. The STM bias voltage is referred to the sample and all the images were obtained in constant current mode. The $\mathrm{Au}(111)$ single crystal was prepared by repeated cycles of sputtering $\left(\mathrm{Ar}^{+}, 1.5 \mathrm{keV}\right)$ followed by thermal annealing at about $700 \mathrm{~K}$. The bis-pyrene was thermo-evaporated from a quartz crucible heated to $530 \mathrm{~K}$ and deposited on the $\mathrm{Au}(111)$ surface for substrate temperatures in the range from $180 \mathrm{~K}$ to room temperature. After deposition the samples were rapidly transferred into the analysis chamber and cooled down to $\mathrm{LN}_{2}$ temperature inside the LT-STM.

\section{Results and discussion}

The optical properties of bis-pyrene in solution and for thick films evaporated under high vacuum conditions on glass substrate have been investigated by UV-Vis spectroscopy. Fig. 2 displays two normalized UV-Vis spectra, which have been recorded for bis-pyrene in solution (blue curve) and for a thick film deposited on BK7 (red curve). In solution its appears that bis-pyrene exhibits a weak positive solvatochromism effect. The compound is transparent above $550 \mathrm{~nm}$, and shows a single absorption band with a maximum at $441 \mathrm{~nm}$. In pyrene-containing compounds this low-energy band can be attributed to the long-axis polarized

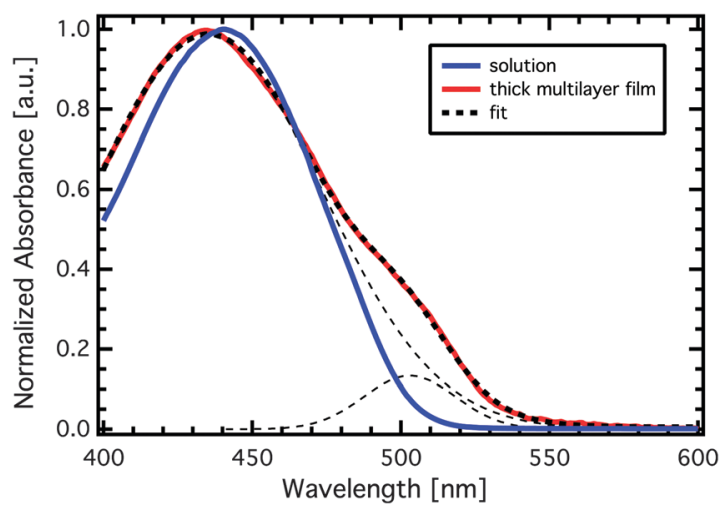

Fig. 2 Normalized UV-vis spectra measured for bis-pyrene in solution in THF (blue curve) and for a thick film deposited under HV conditions (red curve). Notice the pronounced shoulder in the case of the evaporated film. The UV-Vis spectra of the thick film has been fitted by two gaussian curves (dashed line). 
transition ${ }^{1} \mathrm{~L}_{\mathrm{a}} \cdot{ }^{17-19}$ The absorption spectrum of the thick vacuum deposited film on BK7 is composed of an intense transition band (with a maximum at $434 \mathrm{~nm}$, equally attributed to the long-axis polarized transition ${ }^{1} \mathrm{~L}_{\mathrm{a}}$ ) and a shoulder at higher wavelengths. This spectrum can be fitted by two gaussian curves, as displayed in Fig. 2, which allow us to determine the position of the second peak (505 nm). Interestingly, this shoulder has not been observed neither in THF solution nor with other solvents, which suggests that this shoulder arises from an interaction of the transition dipole moment of neighbouring molecules. This phenomenon is well-known as Davydov splitting ${ }^{20}$ in case of organic crystals.

In order to elucidate the origin of this additional absorption band, the coverage-dependent optical properties of bis-pyrene deposited on BK7 substrate have been investigated by S-CRD spectroscopy, which allows to cover the very low coverage regime inaccessible by conventional UV-Vis spectroscopy. The influence of the substrate temperature (RT or $120 \mathrm{~K}$ ) has also been investigated. In Fig. 3a four s-CRD spectra in the wavelength range from $420 \mathrm{~nm}$ to $680 \mathrm{~nm}$ are displayed, which have been recorded for molecular densities from 1.6 to $6.6 \times 10^{13} \mathrm{~cm}^{-2}$ (for the details of the data treatment refer to the ESI, $\dagger$ Fig. S2). Depending on the molecular density s-CRD spectra show distinct features.

The spectra of the two smallest coverages show an intense transition band at $434 \mathrm{~nm}$ and the compound is transparent above $550 \mathrm{~nm}$ (similar to the UV-Vis in THF except for the slight blue shift). At coverages of $4.6 \times 10^{13} \mathrm{~cm}^{-2}$ and above the spectra show the same features as in the case of the UV-Vis recorded for thick films deposited on glass: an intense transition band at $434 \mathrm{~nm}$, a shoulder around $505 \mathrm{~nm}$, and the compound is transparent above $550 \mathrm{~nm}$. The loss per pass at $434 \mathrm{~nm}$ is linearly proportional to the molecular density, as expected (see ESI, $\dagger$ Fig. S3).

In order to visualize the intensity changes at $505 \mathrm{~nm}$, which had been found ex situ for the thick film, the normalized spectra in the range of 480 to $540 \mathrm{~nm}$, which is covered by a single set of highly reflective mirrors, are displayed in Fig. $3 \mathrm{~b}$ for molecular densities ranging from $1.6 \times 10^{13}$ to $1.2 \times 10^{14} \mathrm{~cm}^{-2}$. We can clearly observe an increase of the relative absorption around $505 \mathrm{~nm}$ as a function of coverage up to a coverage of $6.6 \times 10^{13} \mathrm{~cm}^{-2}$. For higher film thicknesses the intensity of this shoulder remains constant despite the intensity increase of the peak at $434 \mathrm{~nm}$. The shoulder can thus be attributed to the interaction of the transition dipole moment of the molecules. It is known that we can observe the appearance of spectral components at low and high energy in the absorption spectrum of chromophores coupled in different geometries. ${ }^{21}$ This effect is well-known in the case of molecular crystals ${ }^{20}$ and has been observed in the case of the formation of amorphous films with crystal-like structure at very short range. ${ }^{22,23}$ The shoulder is already visible at a coverage of $3.1 \times 10^{13} \mathrm{~cm}^{-2}$, which corresponds approximatively to the completion of the first monolayer as determined by estimating the area covered by a single bis-pyrene molecule assuming that it lies flat on the surface. We attribute the development of this shoulder to the formation of molecular islands with particular molecular stacking allowing the interaction
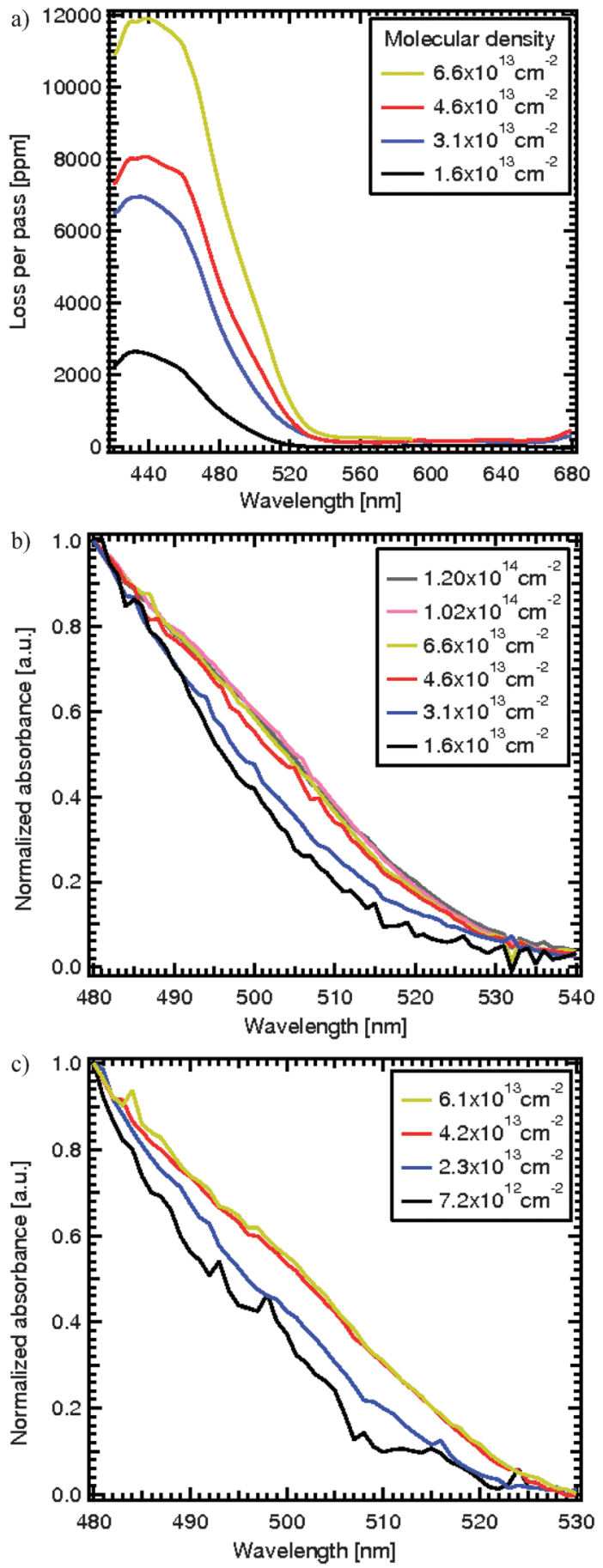

Fig. 3 (a) S-CRD spectra recorded for bis-pyrene deposited on BK7 at $120 \mathrm{~K}$ for various molecular density. (b) Raw data recorded in the range 480-540 nm (single set of mirrors), normalized at $480 \mathrm{~nm}$ highlighting the appearance of the shoulder around $505 \mathrm{~nm}$. (c) The same process used in the case of the film deposited at RT.

of transition dipole moments of the first and the second layer before the completion of the first ML. At higher coverages we observe that the intensity of this shoulder saturates for molecular density above $4.6 \times 10^{13} \mathrm{~cm}^{-2}$. This suggests that above this coverage the interfacial region between the first layer and the following layers does not increase any more. 
Similar results are obtained for bis-pyrene films deposited on BK7 at room temperature. Full s-CRD spectra recorded in the wavelength range from 420 to $680 \mathrm{~nm}$ are shown in the ESI $\dagger$ (Fig. S4). Here again, only the molecular transition at $434 \mathrm{~nm}$ is observed for coverages up to $2.3 \times 10^{13} \mathrm{~cm}^{-2}$, and the shoulder at $505 \mathrm{~nm}$ appears for coverages above $4.2 \times 10^{13} \mathrm{~cm}^{-2}$. The normalized absorbance at various molecular densities recorded in the range 480-530 $\mathrm{nm}$ are shown in Fig. 3c. As in the case of the deposition at RT, an intensity increase of the transition at $505 \mathrm{~nm}$ is observed between $7.2 \times 10^{12} \mathrm{~cm}^{-2}$ and $2.3 \times 10^{13} \mathrm{~cm}^{-2}$, indicating that the interaction of molecules in the first and the second layer already takes place at this low coverage. A saturation of the normalized absorption is observed above $4.2 \times 10^{13} \mathrm{~cm}^{-2}$, which can again be explained by an interaction of the transition dipole moments of each molecules. Note that the saturation of the shoulder takes place at lower molecular density in this case compared to a deposition at LT, which will be discussed below.

In order to get a better understanding of the changes observed with s-CRD we have undertaken complementary STM investigations of the morphology of thin bis-pyrene films. For simplicity we have chosen the $\mathrm{Au}(111)$ surface as a substrate because it is wellknown that this surface interacts weakly with organic adsorbates. ${ }^{24}$ This gives us the possibility to study the physisorbed bis-pyrene molecules in a weakly perturbed state, which should be similar to the state on BK7 substrate used for the optical measurements. STM images were recorded for bis-pyrene deposited on $\mathrm{Au}(111)$ surface as function of the coverage and the temperature.

We observed that the growth process until the completion of the first ML is mainly independent of the substrate temperature in the range $210 \mathrm{~K}$ to $\mathrm{RT}$. Only the size of the domains is affected: the lower the deposition temperature, the smaller the domain size. Interestingly two two-dimensional structures are observed for coverages between 0.5 ML and completion of the first ML, whereas, only one of these domains is observed for coverages above the ML. The early stages of the molecular growth (up to the monolayer formation) will be detailed in a future publication. Fig. 4a shows a STM image of 1.1 ML of bispyrene deposited on $\mathrm{Au}(111)$ at RT. The first ML is highly organized as shown in the insert where the very high resolution allows us to distinguish pyrene moieties and octyloxy chain connected to the central benzene ring. Bis-pyrene molecules lie flat on the surface, with the plane formed by pyrene moieties and oligophenylene-vinylene bridge parallel to the surface and the octyloxy chain are oriented perpendicularly with respect to the axis of the conjugated system (Pyr-OPV-Pyr). The unit cell has the following parameters: $a=3.30 \pm 0.2 \mathrm{~nm} ; b=1.95 \pm$ $0.2 \mathrm{~nm}$ and $\theta=90 \pm 2^{\circ}$ and contains two molecules. The unit cell vector a (resp. b) run along the [110] (resp. [112]) direction. The density of this first ML, calculated from the unit cell parameters, is $3.1 \times 10^{13}$ molecules per $\mathrm{cm}^{2}$, which is in very good agreement with the estimation of the molecular density of the first ML from the CRD results. The apparent height of the molecule in the first ML (and also before the completion of this first ML) is $160 \mathrm{pm}$ and is independent of the bias voltage. We can observe that the second ML starts to grow in a disordered manner and preferentially at places where defects are localized

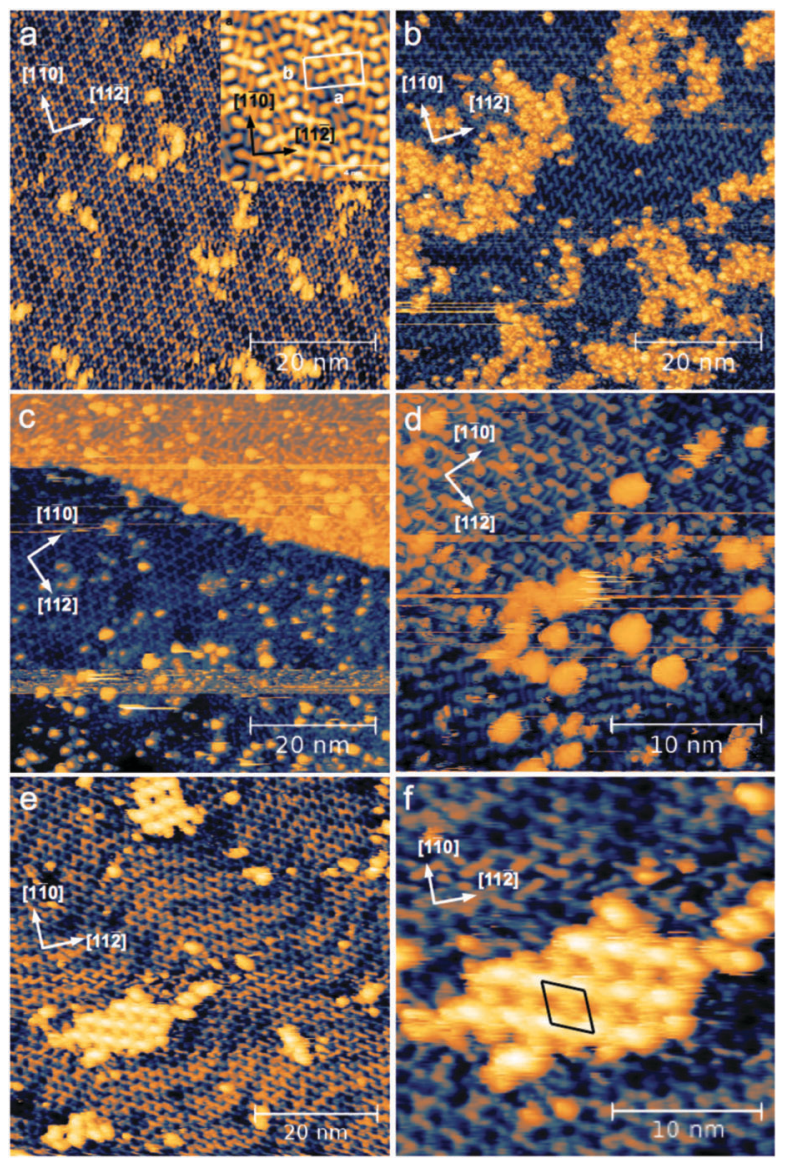

Fig. 4 STM images of bis-pyrene deposited on $A u(111)$ at different temperatures and coverages. (a) RT, $1.1 \mathrm{ML}, \mathrm{Ub}=1 \mathrm{~V}$, It $=40 \mathrm{pA}$. Inset: structure of the highly organized first monolayer. (b) RT, $1.5 \mathrm{ML}, \mathrm{Ub}=-1 \mathrm{~V}$, It $=50 \mathrm{pA}$. (c) and (d) $180 \mathrm{~K}, 1.1 \mathrm{ML}, \mathrm{Ub}=0.9 \mathrm{~V}$, It $=50 \mathrm{pA}$. (e) and (f) $1.1 \mathrm{ML}$ deposited at $180 \mathrm{~K}$ and annealed at RT for $150 \mathrm{~min}$. Ub $=-0.5 \mathrm{~V}$, It $=70 \mathrm{pA}$.

in the first ML or at the domains boundaries, indicating that molecules deposited on top of the first ML are able to diffuse at this temperature. The STM image in Fig. $4 \mathrm{~b}$ has been recorded for a coverage of 1.5 ML. The previous observations are confirmed: the second ML grows in a disordered manner, forming amorphous islands on top of a highly ordered first ML. The apparent height of the amorphous structure is between $200-250 \mathrm{pm}$, which suggests that molecules have a different orientation than in the first layer.

Fig. 4c presents the STM image of bis-pyrene molecules deposited on a cooled gold substrate $(180 \mathrm{~K})$ at a coverage of 1.1 ML. Here the first ML possesses larger number of defects compared to films deposited at temperatures above $210 \mathrm{~K}$. Moreover, the molecules on top of this first ML have apparent heights in the range $200-250 \mathrm{pm}$ and $350-400 \mathrm{pm}$. According to the previous apparent height measurements, this can be explained by the adsorption of single molecules not lying strictly flat and the formation of molecular structures consisting of two molecules, on top of each other. A closer look at the images (Fig. 4d) reveals that deposition at temperatures below $210 \mathrm{~K}$ still leads to formation of a highly organised first ML, where the molecule lies flat on the surface. However, here the first ML is 
composed of three different patterns which is in contrast to films deposited at temperatures above $210 \mathrm{~K}$ where only one of the patterns was observed at a time. The main difference between these patterns is the spatial arrangement of the pyrene moieties inside the molecule (cis or trans configuration) and the interdigitation of the alkyl chains.

Annealing the STM sample for 150 minutes at room temperature under UHV conditions leads to a partial reconstruction of the first ML: one of the above mentioned patterns vanish, and larger domains showing the structure displayed in the insert of Fig. 4a are observed (Fig. 4e). Interestingly, the molecules on the second layer also reorganise and form a new ordered structure. A higher resolution image of this structure is displayed in Fig. 4f. The apparent height of this structure is $250 \mathrm{pm}$ and the unit cell parameters are $2.7 \pm 0.2 \mathrm{~nm} ; 2.9 \pm 0.2 \mathrm{~nm} ; \theta=114 \pm 2^{\circ}$. Unfortunately the resolution is not sufficient to discern bis-pyrene molecules constituting this structure but it clearly shows that an organized growth takes place. Interestingly this structure has not been observed in the case of RT deposition. This implies that diffusion in the second monolayer is apparently slow so that a certain time is need for self-organisation. For deposition temperatures close to RT the self-organisation has not been observed because of a rapid sample transfer into the STM where the surface was quenched to LN temperatures. The disorder in the second layer can thus be explain by the kinetics of diffusion at room temperature. The STM observations clearly indicate that the monolayer density of a self-organized layer of bis-pyrene in flat configuration on $\mathrm{Au}(111)$ is $3.1 \times 10^{13} \mathrm{~cm}^{-2}$, independently of the temperature.

In the case of the s-CRD measurement we observe that particular interaction of the molecular transition dipole moment, which is attributed to the formation of the second layer, already starts below this molecular density. This strongly suggests the formation of molecular multilayers allowing these kind of interactions before a completion of the first monolayer. The saturation of the signal for molecular density of $4.2 \times 10^{13} \mathrm{~cm}^{-2}$, in the case of the deposition performed at RT, corresponds approximatively to a coverage of 1.35 ML observed in STM. This saturation of the signal can be explain considering different growth modes depending on the surface. On $\mathrm{Au}(111)$ we observed a growth mode with a completion of the first ML happening before the second layer starts to grow, independently of the temperature. Since higher coverages have not been investigated this could either be a Frank-van der Merwe or Stranski-Krastanov growth mode (see Fig. 5) for the RT deposition. At LT, the mode can clearly be identified as being of Stranski-Krastanov type, which is indicated by the presence of structures in the STM images showing an apparent height of two times the apparent height measured for the second ML. On BK7, the appearance of the signal at coverages below the ML and the saturation of the signal at coverages below an equivalent of 2 ML suggests strongly a Volmer-Weber growth mode that is contrary to the growth mode observed on $\mathrm{Au}(111)$. On BK7 no complete wetting takes place. As underlined before, the saturation of the signal takes place at lower coverages in the case of deposition performed at RT. This can be explain by kinetics.

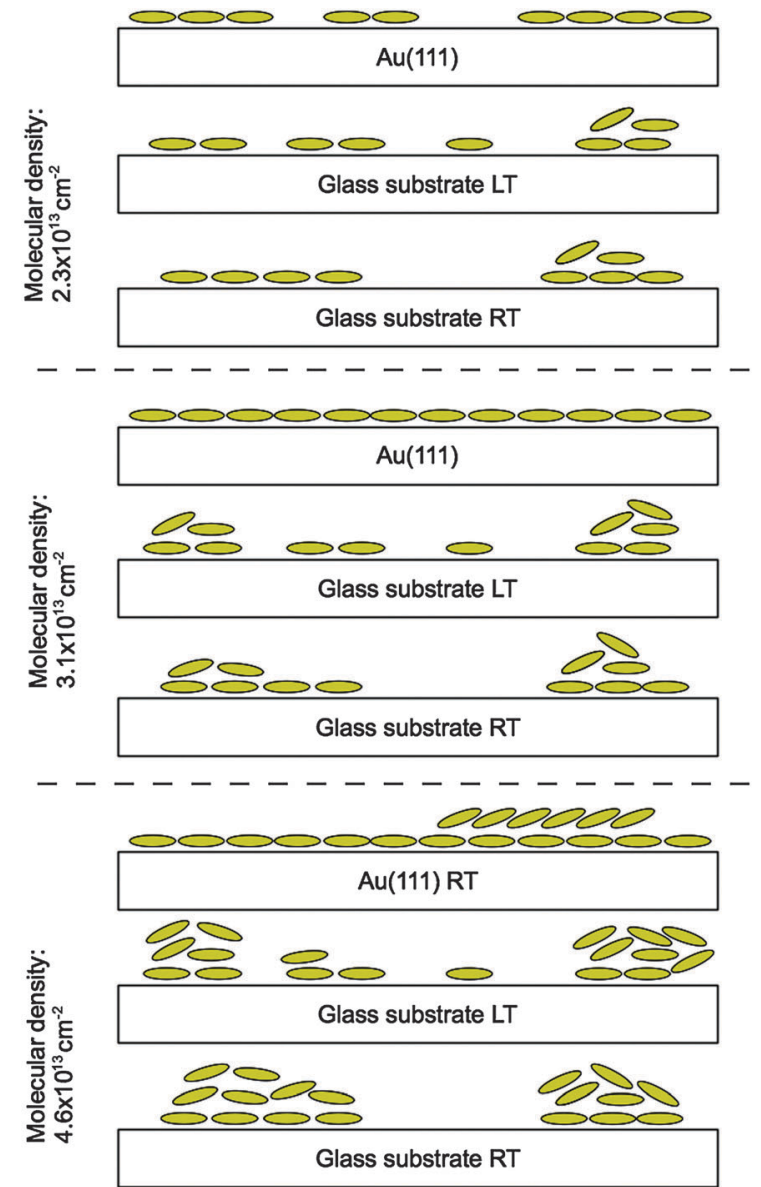

Fig. 5 Schematic representation of bis-pyrene growth on Au(111) and BK7 at RT and LT.

Fig. 5 displays schematic growth processes proposed for bispyrene on $\mathrm{Au}(111)$ and on BK7 (at RT and LT). It is well-know ${ }^{25}$ that in the case of thin film growth, the number of molecular islands strongly depends on the substrate temperature. Increasing the temperature leads to an enhanced diffusion, which favours the growth of larger molecular islands. This temperature effect leads also to a decrease of the number density of islands at higher temperature. The distinct evolution of the shoulder at $505 \mathrm{~nm}$ in S-CRD measurements in the submonolayer regime can thus be explained by the different growth kinetics at LT and RT, i.e. the formation of fewer molecular islands at RT. The growth mode corresponds however to a Volmer-Weber type at the two temperatures as it is shown in Fig. 5 .

In order to investigate the electronic states of the adsorbed bis-pyrene molecules on $\mathrm{Au}(111)$ we have undertaken scanning tunnelling spectroscopy measurements. Local $I(V)$ measurements were performed on top of the pyrene groups and averaged (blue line) in order to reduce the noise (see Fig. 6). The differential conductivity $\mathrm{d} I / \mathrm{d} V$, which corresponds to the local density of states (LDOS) of the sample, has then been calculated from the $I(V)$ curve by numerical differentiation (red curve). The spectra clearly show band gap with a residual conductivity in the gap, which is due to the $\mathrm{Au}(111)$ substrate. The $\mathrm{d} I / \mathrm{d} V$ curve shows 


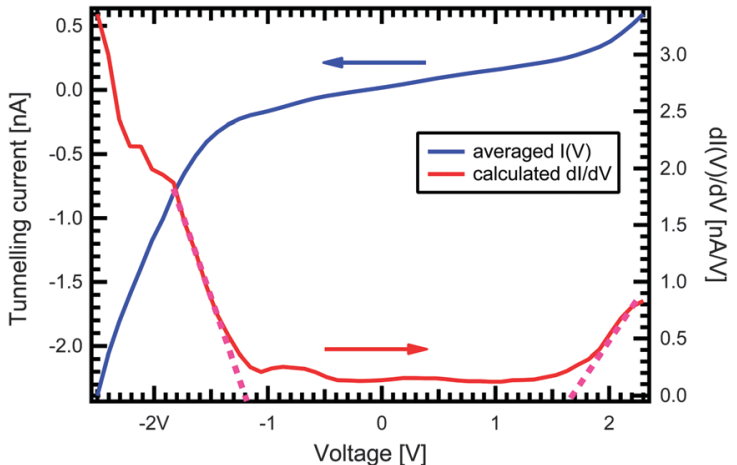

Fig. 6 Averaged local I( $V$ ) curves (blue) measured for bis-pyrene on $\mathrm{Au}(111)$. An optical gap of $2.85 \mathrm{eV}$ could be determined from the calculated $\mathrm{d} / / \mathrm{d} V$ curve (red).

clearly a HOMO-LUMO gap, which is indicated by the steep increase of the LDOS below $-1.2 \mathrm{eV}$ (HOMO) and above $1.65 \mathrm{eV}$ (LUMO). The thus observed gap of $2.85 \mathrm{eV}$ is in excellent agreement with the optical gap, which corresponds to the transition at $434 \mathrm{~nm}(2.86 \mathrm{eV})$ in the s-CRD spectra. This suggests that the interaction of the bis-pyrene with the $\mathrm{Au}(111)$ surface is indeed weak and thus very similar to the adsorption on the BK7 substrate used for the s-CRD measurements. The small peaks, which are observed in the region $-1 \mathrm{eV}$ to $-0.5 \mathrm{eV}$, can be attributed to the Shockley surface band of the $\mathrm{Au}(111)$ surface, which is located near $-0.5 \mathrm{eV}^{26}$ on the clean surface. It has been reported that this surface state is shifted by the presence of adsorbates, ${ }^{24,27}$ which seems also be the case here.

\section{Conclusions}

We have investigated the optical properties of the 1,4-di- $n$ octyloxy-2,5-bis(pyren-1-ylethenyl)benzene by the means of s-CRD spectroscopy and the influence of the temperature during growth process by STM. Combining a highly sensitive surface spectroscopic method (s-CRD) with STM investigations provides the possibility to elucidate the optical and morphological properties of thin molecular layers. S-CRD measurements indicate for low submonolayer densities that bis-pyrene molecules possess optical properties which are very similar to those observed in solution. This suggests that in the low submonolayer range the optical transitions are neither affected by intermolecular nor by molecule substrate interactions. At coverages below the monolayer density the appearance of the spectral component at $505 \mathrm{~nm}$ is attributed to the formation of multilayer islands and the saturation of the signal to the completion of the second molecular layer of these islands, which depend of the temperature due to growth kinetics. Our analysis shows that the shoulder is the result of interlayer interaction of the transition dipole moment of molecules of the emerging second layer with the molecules from the first layer. STM also reveals that the deposition temperature also plays a very important role on the growth process of the second monolayer: while an amorphous structure is observed in the case of depositions performed at low temperature, the formation of an organized structure is observed for films grown at room temperature. Furthermore, it has been shown that the molecular film wets the $\mathrm{Au}(111)$ substrate, which is not the case for the BK7 substrate. Finally the band gap of $2.85 \mathrm{eV}$, which has been determined by scanning tunnelling spectroscopy is in very good agreement with the optical transition observed at $434 \mathrm{~nm}$ in the s-CRD spectra, which corroborates the weak interaction of the molecules with these substrates.

\section{Acknowledgements}

The authors gratefully acknowledge the support by the European Research Council through an Advanced Research Grant (246645ASC3), the DFG through the project (HE 3454/21-1), and the support by the UFA-DFH through the project PhD7-12.

\section{Notes and references}

1 A. Facchetti, Chem. Mater., 2011, 23, 733.

2 A. Mishra, C.-Q. Ma and P. Bäuerle, Chem. Rev., 2009, 109, 1141.

3 M. Iyoda, M. Hasegawa and Y. Miyake, Chem. Rev., 2004, 104, 5085.

4 E. Menard, M. A. Meitl, Y. Sun, J.-U. Park, D. Jay-Lee Shir, T.-S. Nam, S. Jeon and J. A. Rogers, Chem. Rev., 2007, 107, 1117.

5 J. E. Anthony, Chem. Rev., 2006, 106, 5028.

6 N. Koch, ChemPhysChem, 2007, 8, 1438.

7 T. M. Figueira-Duarte and K. Müllen, Chem. Rev., 2011, 111, 7260.

8 F. Moggia, C. Videlot-Ackermann, J. Ackermann, P. Raynal, H. Brisset and F. Fages, J. Mater. Chem., 2006, 16, 2380.

9 Electronic devices based on bis-pyrene are under investigation.

10 A. Kartouzian, M. Thämer, T. Soini, J. Peter, P. Pitshi, S. Gilb and U. Heiz, J. Appl. Phys., 2008, 104, 124313.

11 M. Thämer, A. Kartouzian, P. Heister, S. Gerlach, M. Tschurl, U. Boesl and U. Heiz, J. Phys. Chem. C, 2012, 116, 8642.

12 S. Gilb, K. Hartl, A. Kartouzian, J. Peter, U. Heiz, H.-G. Boyen and P. Ziemann, Eur. Phys. J. D, 2007, 45, 501.

13 M. Thämer, A. Kartouzian, P. Heister, T. Lünskens, S. Gerlach and U. Heiz, Small, 2014, 10, 2340.

14 T. Lünskens, P. Heister, M. Thämer, C. A. Walenta, A. Kartouzian and U. Heiz, Phys. Chem. Chem. Phys., 2015, 17, 17541.

15 A. Kartouzian, P. Heister, M. Thämer, S. Gerlach and U. Heiz, J. Opt. Soc. Am. B, 2013, 30, 541.

16 A. Kartouzian, M. Thämer and U. Heiz, Phys. Status Solidi B, 2010, 247, 1147.

17 S. Leroy-Lhez, M. Allain, J. Oberlé and F. Fages, New J. Chem., 2007, 31, 1013.

18 H. Li, D. R. Powell, R. K. hayashi and R. West, Macromolecules, 1998, 31, 52.

19 S. Leroy Lhez, A. Parker, P. Lapouyade, C. Belin, L. Ducasse, J. Oberle and F. Fages, Photochem. Photobiol. Sci., 2004, 3, 949.

20 A. S. Davydov, Zh. Eksp. Teor. Fiz., 1948, 18, 210. 
21 M. Kasha, Radiat. Res., 1963, 20, 55.

22 Y. Maruyama and N. Iwasaki, Chem. Phys. Lett., 1974, 24, 26.

23 R. Jankowiak, K. D. Rockwltz and H. Bässler, J. Phys. Chem., 1983, 87, 552.

24 N. Nicoara, E. Roman, J. M. Gomez-Rodrigez, J. A. MartinGago and J. Mendez, Org. Electron., 2006, 7, 287.
25 J. A. Venables, G. D. T. Spiller and M. Hanbücken, Rep. Prog. Phys., 1984, 47, 399.

26 W. Chen, V. Madhavan, T. Jamnela and M. F. Crommie, Phys. Rev. Lett., 1998, 80, 1469.

27 T. Andreev, I. Barke and H. Hövel, Phys. Rev. B: Condens. Matter Mater. Phys., 2004, 70, 205426. 